\title{
Sliding Mode Output Feedback Control of a Flexible Rotor Supported by Magnetic Bearings
}

\author{
A.S. LEWIS ${ }^{\mathrm{a}, *}$, A. SINHA ${ }^{\mathrm{b}}$ and K.W. WANG ${ }^{\mathrm{b}}$ \\ ${ }^{a}$ The Pennsylvania State University Applied Research Lab, State College, PA 16804, USA; \\ ${ }^{\mathrm{b}}$ Mechanical Engineering Department, The Pennsylvania State University, University Park, PA 16802, USA
}

(Received 6 August 1999; In final form 16 September 1999)

\begin{abstract}
A new sliding mode feedback algorithm is applied to control the vibration of a flexible rotor supported by magnetic bearings. It is assumed that the number of states is greater than the number of sensors. A mathematical model of the rotor/magnetic bearing system is presented in terms of partial differential equations. These equations are then discretized into a finite number of ordinary differential equations through Galerkin's method. The sliding mode control law is designed to be robust to rotor unbalance and transient disturbances. A boundary layer is introduced around each sliding hyperplane to eliminate the chattering phenomenon. The results from numerical simulations are presented that not only corroborate the validity of the proposed controller, but also show the effects of various control parameters as a function of the angular speed of the rotor. In addition, results are presented that indicate how the current required by the magnetic bearings is affected by control parameters and the angular speed of the rotor.
\end{abstract}

Keywords: Magnetic bearings, Sliding mode control, Flexible rotor, Rotor unbalance, Vibration

\section{INTRODUCTION}

Rotor vibration has been an important problem since the development of turbomachinery and electrical machines as indicated by Vance (1988), Dimarogonas and Paipetis (1983), and LaLanne and Ferraris (1990). One of the key sources of vibration is the inevitable rotor unbalance and the resulting dynamic force that is proportional to the square of the rotor speed. Hence, rotor vibration is a more severe problem for machines operating at higher speeds. In fact, for good performance at higher rotational speeds, it is necessary to have amplitudes of vibration smaller than those for the same performance at lower speeds as shown by Rao (1995). One approach that has been taken to reduce the severity of rotor unbalance is rotor balancing, a technique that essentially adds a mass in such a position that it cancels the effect of the unbalance. However, this is not a straightforward process

*Corresponding author. E-mail: ASL103@email.psu.edu. 
since the amount and location of the eccentric mass are not easily determined in a real machine. Further, this approach does not completely eliminate all the effects of the unbalance and cannot adapt to unexpected transient disturbances. Consequently, active control of rotor vibration has been considered in the literature.

Actuators that have been used to control rotor vibration include piezo-electric pusher plates by Palazzolo et al. (1989), magnetic bearings by Ulbrich and Anton (1984), and fluid film pressure control. Fluid film bearings, which usually have a long life, require a constant oil supply and can become easily contaminated. Pusher plates, though adaptable to various shaft-loading positions, have contact with the shaft that leads to wear problems. Further, Bobbert and Schamhardt (1990) have shown that it is difficult to accurately calculate the application point of the force. Conversely, magnetic bearings require no lubrication and do not contact the shaft thus avoiding wear problems. Hence, a significant amount of research has been devoted to develop a highly reliable magnetic bearing system for active vibration control.

It is well known that the attractive force of each magnetic bearing varies proportionally to the square of the coil current and is inversely proportional to the square of the airgap between the rotor and stator. As a result, when the airgap decreases, the attractive force increases for a fixed coil current and the object is brought closer and closer to the magnet. Conversely, when the airgap increases, the object is continuously drawn away from the magnet. This phenomenon is known as negative stiffness and results in an inherently unstable system. Thus, for a stable configuration, active control must be employed. An active suspension system continuously monitors the object's position and applies a force to return the system to a stable configuration. Some details of magnetic bearing design, in particular the integration of displacement and velocity sensors within the magnetic bearing, were discussed by Ulbrich and Anton (1984). Sinha (1990) has summarized the basic issues associated with the stability of active magnetic suspension systems. In addition, Humphris et al. (1986) reported the effects of control algorithms on magnetic bearing stability. More specifically, they studied differences in electronic circuit bandwidths, sensor positioning, and the overall bearing stiffness and damping coefficients. Allaire et al. (1986) investigated critical speeds and unbalance response of a flexible rotor in magnetic bearings. Modal control of a flexible rotor was demonstrated by Salm and Schweitzer (1984).

One of the most important problems involved in the control of rotors is the rotor unbalance caused by the unavoidable uncertainties in the rotor eccentricity. Okada (1994) combined local proportional-derivative (PD) feedback with bending force feedback to each magnet in a disturbance based observer for a flexible rotor. Herzog et al. (1996) have investigated unbalance compensation using generalized notch filters in conjunction with multivariable feedback but they do not design a specific controller. Lum et al. (1996) employed a technique called adaptive autocentering control that compensates for transmitted force due to unbalance. They accomplish their objective by obtaining on-line estimates of the rotor unbalance properties and using these estimates to compute the mass center position and velocity, which are unknown and cannot be measured directly. Knospe and Tamer (1997) studied robust adaptive control of unbalance response for a flexible rotor by synthesizing five controllers. Based on the works above, it is apparent that algorithms that can be designed to compensate for rotor uncertainties are advantageous. Although quantifying the exact amount and location of the unbalance is quite difficult, it is generally a straightforward process to establish an upper bound of the unbalance force. Sliding mode control theory, for example, Utkin (1977, 1983), can be designed for robustness with respect to uncertainties and transient disturbances provided their bounds are known. In particular, an a priori bound of the output response can be determined knowing the bounds of the disturbance, which is not necessarily true with a proportional-integralderivative (PID) controller. 
Sinha et al. (1991) have considered sliding mode control of a rigid rotor supported by magnetic bearings. However, many rotor systems cannot be treated as rigid and flexible effects must be taken into account. Rundell et al. (1996) used a sliding mode observer and controller to stabilize the rotational motion of a vertical shaft magnetic bearing. To develop their controller, they needed estimates of the derivatives of the disturbance. They also used a matrix to help account for robustness and gave an example of a matrix computed experimentally, but they did not present a procedure for determining the matrix on a general basis. Tian and Nonami (1996) applied discrete-time sliding mode control to a flexible rotor/magnetic bearing system. They presented the design of a robust observer requiring several matrices to exist, but they did not give methods for finding these matrices. Nonami and Ito (1996) investigated $\mu$-synthesis of a flexible rotor/magnetic bearing system. However, this approach involves a complicated iterative and nonconvex numerical procedure. This paper explores a new approach to the robust control of a flexible rotor using sliding mode control with only output feedback.

Using the upper bound of the unbalance force, which is a function of mass unbalance and eccentricity, a controller is to be developed that will guarantee a specified performance level in the presence of uncertainties in the eccentricity and unbalance mass as long the unbalance force is under this bound. Robustness in this context is to be interpreted to indicate that the eccentricity and unbalance mass do not have to be known exactly to guarantee an acceptable performance level. It is a straightforward process to guarantee robustness with sliding mode control if all the states are known, which is a good assumption for a rigid rotor. In the context of active control of flexible rotors via magnetic bearings, the number of effective actuators and sensors is generally small not only when compared to the dimension of the full-order model, but also to the number of the states of the reducedorder model. As a result, not all the states are available for measurement. It is found that the design of the sliding mode controller that must be developed on the basis of outputs requires the upper bounds of the states. This is a coupled problem since the states are influenced by the controller and the bounds on the states are required to design the controller. Yallapragada et al. (1996) and Wang and Fan (1994) have examined sliding mode controllers based on output feedback. Recently, Lewis and Sinha $(1999,1995)$ have addressed this problem with a novel sliding mode control output feedback scheme for a mechanical system.

This paper presents an application of the control methodology formulated by Lewis and Sinha (1999, 1995) for the control of a flexible rotor via magnetic bearings. The model of the rotor system is developed through Hamilton's principle and a technique is given to reduce the order of the model for control law development. Numerical computations, which determine the gains necessary to ensure robustness to uncertainties in the rotor eccentricity, are presented to corroborate the applicability of the approach to the rotor problem. Also, results are presented to show the effects of control parameters as they relate to steady-state amplitude, current requirements of the magnetic bearings, and changes in the rotational speed.

\section{DEVELOPMENT OF THE FLEXIBLE ROTOR MODEL}

Figure 1 shows a schematic of a flexible rotor system with one disk. The system equations of

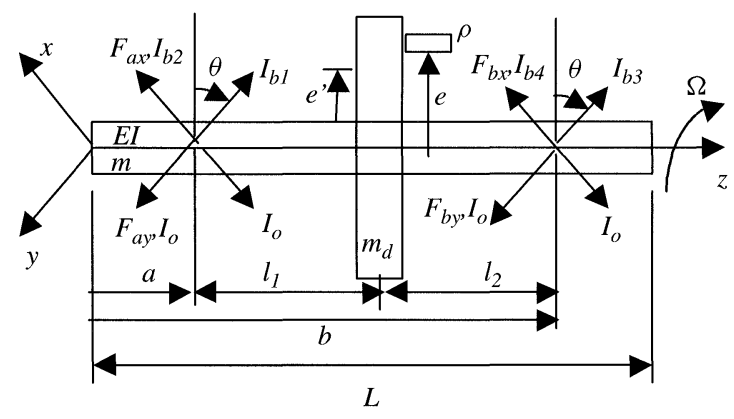

FIGURE 1 Rotor model and bias currents 
motion are based on the following assumptions:

(a) In addition to the main disk shown in Fig. 1, there are two other disks on the shaft that have no unbalance.

(b) Transverse vibration can occur simultaneously in the $x$-and $y$-directions.

(c) The rotary inertia and shear deformation of the shaft are negligible since the shaft is long and thin.

(d) The eccentricity $e^{\prime}$ (distance between geometric center of the rotor and overall rotor mass center) is small.

(e) The unbalance mass is small compared to the mass of the disk.

(f) Deflections of the shaft are small.

(g) Damping is proportional to the stiffness.

Using Hamilton's principle, two partial differential equations describing motion in the $x$ - and $y$ directions are derived. A discretized system model is obtained by Galerkin's method using the eigenfunctions of a free-free Euler-Bernoulli beam as comparison functions, Lewis (1994). The discretized equations of motion are

$$
\bar{M} \ddot{r}+\left(\bar{G}+C_{\mathrm{D}} \bar{K}\right) \dot{r}+\bar{K} r=\bar{B} F_{\mathrm{c}}+\bar{D} F_{\mathrm{d}}(t)
$$

where

$$
\begin{aligned}
& r=\left[p_{1}, p_{2}, \ldots, p_{N}, q_{1}, q_{2}, \ldots, q_{N}\right]^{\mathrm{T}} \\
& F_{\mathrm{c}}=\left[\begin{array}{llll}
F_{a x} & F_{a y} & F_{b x} & F_{b y}
\end{array}\right]^{\mathrm{T}} \\
& F_{\mathrm{d}}(t)=\rho e \Omega^{2}\left[\begin{array}{ll}
\cos \Omega t & \sin \Omega t
\end{array}\right]^{\mathrm{T}}
\end{aligned}
$$

and $C_{\mathrm{D}}$ is a constant selected to provide a specified damping ratio. The mass matrix $\bar{M}$ is symmetric and positive definite. The gyroscopic matrix $\bar{G}$ is skew-symmetric. The stiffness matrix $\bar{K}$ is symmetric and positive semidefinite. $F_{a x}$ and $F_{b y}$ are the net forces at the left end of the shaft in the $x$ - and $y$-directions, respectively. Similarly, $F_{b x}$ and $F_{b y}$ are the net forces at the right end of the shaft. Relationships between these forces and currents in the magnetic bearing actuators can be found in the
Appendix. The $p_{i}$ and $q_{i}$ are generalized coordinates and $N$ is the number of terms in the Galerkin expansion. The rotor unbalance is $\rho e \Omega^{2}$ where $\rho$ is the mass of the unbalance, $e$ is the eccentricity, and $\Omega$ the angular velocity of the shaft.

In this paper, control algorithms are developed based on output feedback. From Fig. 1, the sensors are located at two axial locations along the shaft, namely at $z=a$ and and $z=b$, providing both position and velocity feedback. Based on this, two output vectors, one for position and one for velocity, can be formed as

$$
\begin{gathered}
Y_{1}=\left[y_{1}, y_{2}, y_{3}, y_{4}\right]^{\mathrm{T}}=C r \\
\dot{Y}_{1}=Y_{2}=\left[\dot{y}_{1}, \dot{y}_{2}, \dot{y}_{3}, \dot{y}_{4}\right]^{\mathrm{T}}=C \dot{r}
\end{gathered}
$$

where $y_{1}$ and $y_{2}$ are the displacements in the $x$ - and $y$-directions, respectively, on the left end of the shaft, $y_{3}$ and $y_{4}$ are displacements in the $x$ - and $y$ directions, respectively, on the right end of the shaft, and $C=\bar{B}^{\mathrm{T}}$ is a $4 \times 2 N$ matrix.

\section{Reduced-Order Model}

Since it is not practical to implement a large order controller, it is necessary to reduce the order of the model to that of a manageable size. In the normal modes approach, the eigenvectors of $\bar{M}^{-1} \bar{K}$, the undamped system without gyroscopic effects, are considered in the following expansion:

$$
r=\Phi \zeta=\sum_{j=1}^{2 n<2 N} \Phi_{j} \zeta_{j}
$$

where $\Phi$ is the mass-normalized $2 N \times 2 n$ matrix of the retained modes or eigenvectors $\Phi_{j}, \zeta$ is the $2 n \times 1$ vector containing the reduced-order coordinates $\zeta j$. Using Eq. (7), the reduced-order system is obtained from Eq. (1) as

$$
\left[\begin{array}{ll}
\dot{X}_{1}^{\mathrm{T}} & \dot{X}_{2}^{\mathrm{T}}
\end{array}\right]^{\mathrm{T}}=\left[\begin{array}{ll}
\dot{\zeta}^{\mathrm{T}} & \ddot{\zeta}^{\mathrm{T}}
\end{array}\right]^{\mathrm{T}}=\dot{X}=A X+B F_{\mathrm{c}}+D F_{\mathrm{d}}
$$


where

$$
\begin{aligned}
& A=\left[\begin{array}{cc}
0 & I \\
-\Phi^{\mathrm{T}} \bar{K} \Phi & -\Phi^{\mathrm{T}}\left(\bar{G}+C_{\mathrm{D}} \bar{K}\right) \Phi
\end{array}\right] \\
& B=\left[\begin{array}{c}
0 \\
\Phi^{\mathrm{T}} \bar{B}
\end{array}\right] \quad D=\left[\begin{array}{c}
0 \\
\Phi^{\mathrm{T}} \bar{D}
\end{array}\right]
\end{aligned}
$$

Finally, using Eqs. (5) and (6), the output vector $Y$ is written as

$$
\begin{aligned}
{\left[\begin{array}{ll}
Y_{1}^{\mathrm{T}} & Y_{2}^{\mathrm{T}}
\end{array}\right]^{\mathrm{T}}=Y } & =\left[\begin{array}{ll}
C & 0 \\
0 & C
\end{array}\right]\left[\begin{array}{l}
r \\
\dot{r}
\end{array}\right] \\
& =\left[\begin{array}{ll}
C & 0 \\
0 & C
\end{array}\right]\left[\begin{array}{ll}
\Phi & 0 \\
0 & \Phi
\end{array}\right] X=C^{*} X
\end{aligned}
$$

\section{DEVELOPING THE SLIDING MODE CONTROL SYSTEM}

Since there are four inputs, four sliding hyperplanes are defined using the approach developed by Asada and Slotine (1986):

$$
\begin{aligned}
s_{i}= & \left(\frac{\mathrm{d}}{\mathrm{d} t}+\lambda_{i}\right)^{2} \int_{0}^{t} y_{i}(\tau) \mathrm{d} \tau \\
= & \dot{y}_{i}(t)+2 y_{i}(t)+\lambda_{i}^{2} \int_{0}^{t} y_{i}(\tau) \mathrm{d} \tau \\
& i=1,2,3,4
\end{aligned}
$$

where the $\lambda_{i}(i=1,2,3,4)$ are control design parameters and $y_{i}$ is the displacement at the $i$ th output. With a properly designed sliding mode control system, the system states reach a desired manifold (intersection of sliding hyperplanes) and stay on the manifold thereafter. These two stages are referred to as the reaching condition $\left(s_{i} \dot{s}_{i}<0\right)$ and sliding condition, respectively. Using Eqs. (10) and (11),

$$
S=\left[\begin{array}{llll}
s_{1} & s_{2} & s_{3} & s_{4}
\end{array}\right]^{\mathrm{T}}=P Y+\Lambda_{I} Z
$$

where

$$
\begin{aligned}
& P=\left[\begin{array}{ll}
P_{1} & I_{4 \times 4}
\end{array}\right] \\
& P_{1}=\operatorname{diag}\left(\begin{array}{llll}
2 \lambda_{1} & 2 \lambda_{2} & 2 \lambda_{3} & 2 \lambda_{4}
\end{array}\right) \\
& \Lambda_{I}=\operatorname{diag}\left(\lambda_{1}^{2} \lambda_{2}^{2} \lambda_{3}^{2} \lambda_{4}^{2}\right)
\end{aligned}
$$

$Z=$

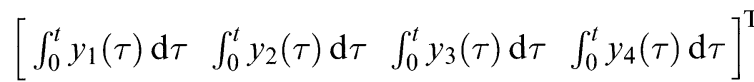

Differentiating Eq. (12), using Eqs. (8) and (10), and noting that $\dot{Z}=Y_{1}$ gives

$$
\dot{S}=P^{*} Y+U+\bar{P} C^{*} A X+\bar{P} C^{*} D F_{\mathrm{d}}(t)
$$

where

$$
\begin{aligned}
P^{*} & =\left[\begin{array}{ll}
\Lambda_{I} & P_{i}
\end{array}\right] \\
\bar{P} & =\left[\begin{array}{ll}
0_{4 \times 4} & i_{4 \times 4}
\end{array}\right] \\
U & =\bar{P} C^{*} B F_{\mathrm{c}}
\end{aligned}
$$

Following the approach developed by Lewis and Sinha $(1999,1995)$, the vector of control forces, $U$, that satisfies both the reaching and sliding conditions is expressed as

$$
U=-\bar{P} C^{*} A \hat{X}-P^{*} Y-K_{G} \bar{S}^{\prime}
$$

where

$$
K_{G}=\operatorname{diag}\left(k_{1} k_{2} k_{3} k_{4}\right)
$$

$$
\bar{S}^{\prime}=\left[\operatorname{sat}\left(\frac{s_{1}}{\phi_{1}}\right) \operatorname{sat}\left(\frac{s_{2}}{\phi_{2}}\right) \operatorname{sat}\left(\frac{s_{3}}{\phi_{3}}\right) \operatorname{sat}\left(\frac{s_{4}}{\phi_{4}}\right)\right]^{\mathrm{T}}
$$

and the $\phi_{i}(i=1,2,3,4)$ are the boundary layer thicknesses used in the saturation function (sat) to eliminate the chattering phenomenon. They are computed by the condition $\phi_{i}=k_{i} / \lambda_{i}$, which has been derived by Asada and Slotine (1986). Proper selection of the diagonal gain matrix $K_{\mathrm{G}}$ insures that the reaching condition $\left(s_{i} \dot{s}_{i}<0\right)$ is satisfied and the system will reach inside the boundary layer. 
It is a function of system states, but not all of the states are available for measurement. This problem is circumvented through proper selection of the estimated state vector, $\hat{X}$.

To illustrate this point, the closed-loop control system is obtained from Eq. (8), noting from Eq. (20) that $F_{\mathrm{c}}=\left(\bar{P} C^{*} B\right)^{-1} U$ and using Eqs. (10) and (21), as

$$
\dot{X}=\tilde{A} X+\tilde{B} \bar{P} C^{*} A \hat{X}+D F_{\mathrm{d}}+\tilde{B} K_{\mathrm{G}} \bar{S}^{\prime}
$$

The estimate of the state vector, $\hat{X}$, is defined as follows:

$$
\dot{\hat{X}}=\left(\tilde{A}+\tilde{B} \bar{P} C^{*} A\right) \hat{X}+\tilde{B} K_{\mathrm{G}} \bar{S}^{\prime} ; \quad \hat{X}(0)=\hat{X}_{0}
$$

where

$$
\tilde{A}=A-B\left(\bar{P} C^{*} B\right)^{-1} P^{*} C^{*} \quad \tilde{B}=-B\left(\bar{P} C^{*} B\right)^{-1}
$$

The choice of $\hat{X}$ dynamics (Eq. (25)) is key to the off-line computation of the control gain vector such that the reaching conditions $\left(s_{i} \dot{s}_{i}<0\right)$ are satisfied. From Lewis and Sinha $(1999,1995)$, the gain vector $K_{\mathrm{v}}$, which is the diagonal of $K_{\mathrm{G}}$ consisting of $\left(k_{1}, k_{2}, k_{3}, k_{4}\right)$, is

$$
K_{\mathrm{v}} \geq\left|\bar{P} C^{*} A\right||X-\hat{X}|+\left|\bar{P} C^{*} D\right|\left|F_{\mathrm{d}}\right|+\eta
$$

This requires the upper bounds of the errors in the state estimate $(X-\hat{X})$ and the dynamic force created by the rotor unbalance. The absolute value signs on the right side of Eq. (27) stand for matrices with absolute values of the elements. The vector $\eta$ consists of small positive constants $\eta_{i}(i=1,2,3,4)$. The upper bound on the magnitude of $(X-\hat{X})$ can now be obtained from Eqs. (24) and (25) as

$$
|X-\hat{X}| \leq\left|\mathrm{e}^{\tilde{A} t}\right|\left|X_{0}-\hat{X}_{0}\right|+\left|\int_{0}^{t} \mathrm{e}^{\tilde{A}(t-\tau)} D F_{\mathrm{d}}(\tau) \mathrm{d} \tau\right|
$$

which is independent of $K_{\mathrm{G}}$. Thus for a given bound on the initial conditions of $X$, the magnitude of $(X-\hat{X})$ can be determined for any time $t$ provided the bound of the unbalance force, which is directly related to the upper bound on the eccentricity, is known. Hence, though the unbalance force and initial conditions may not be known exactly, the reaching condition can still be satisfied as long as the bounds of these quantities are known, which is a reasonable assumption. This analysis can easily be extended to include any bounded transient disturbances.

Inside the boundary layers, Asada and Slotine (1986) have shown that the bound on each output is guaranteed to be

$$
\left|y_{i}(t)\right| \leq 2 \frac{\phi_{i}}{\lambda_{i}}
$$

Note that this type of result is not guaranteed with PID controllers.

\section{COMPUTER SIMULATIONS AND NUMERICAL RESULTS}

The primary focus of this paper is controlling the rotor/magnetic bearing system in the presence of uncertainties in the rotor unbalance. For simplicity, only one mode is used here to illustrate the theory. More modes could be taken since the algorithm with output feedback is still applicable except that the order of the system matrix will increase. The system can be made robust to the uncertainty in the rotor unbalance provided its bound is known. The simulation results, which have been presented here, are computed with both position and velocity feedback. Additional simulations, in which it is assumed that only position measurements are available, have been performed. The control law is implemented digitally with a sampling period of $0.004 \mathrm{~s}$ and the velocities at sensor locations are obtained by difference equations. The results are very similar to those in which it is assumed that the position and velocity are measured directly.

Table I displays the rotor and magnetic bearing parameters used for all numerical calculations. To determine the first flexible mode of the rotor/magnetic bearing system, the full-order model given by Eq. (1) is used. First, it is necessary to determine the number of terms needed in the 
TABLE I Rotor and magnet parameters

\begin{tabular}{ll}
\hline$L=0.4572 \mathrm{~m}$ & $C_{\mathrm{D}}=0.00003$ \\
$m_{\mathrm{d}}=0.618 \mathrm{~kg}$ & $I_{0}=1.0 \mathrm{~A}$ \\
$\rho=0.00588 \mathrm{~kg}$ & $l_{1}=l_{2}=0.102 \mathrm{~m}$ \\
$m=0.558 \mathrm{~kg} / \mathrm{m}$ & $m_{\mathrm{t}}=1.27 \mathrm{~kg}$ \\
$e=0.031 \mathrm{~m}$ & $\mu_{0}=0.000001258 \mathrm{~N} / \mathrm{A}^{2}$ \\
$\eta=0.5 \mathrm{~N} / \mathrm{kg}$ & $E I=83.64 \mathrm{Nm}^{2}$ \\
$h_{0}=0.000508 \mathrm{~m}$ & $N_{\mathrm{p}}=160$ \\
$A_{\mathrm{p}}=0.000146 \mathrm{~m}^{2}$ & $\theta=45^{\circ}$ \\
$a=0.104 \mathrm{~m}$ & $b=0.333 \mathrm{~m}$ \\
\hline
\end{tabular}

Galerkin expansion to achieve an acceptable accuracy level. It is found that $N=10$ provides sufficient accuracy which leads to a full-order state space model with 40 states. The first open-loop critical speed of the rotor based on this model is $6963 \mathrm{rpm}$. With the retention of only the first flexible mode and the rigid body modes, the order of the $A$ matrix in Eq. (8) is $12 \times 12$. Subsequent analyses are based on this reduced-order model.

\section{Verification of Algorithm to Compute Control Gains}

To prove the validity of Eqs. (27) and (28), numerical simulation results are presented for the critical speed of $6963 \mathrm{rpm}(116 \mathrm{~Hz})$. The constants $\eta_{i}$ and $\lambda_{i}$ are taken to be $0.5 \mathrm{~N} / \mathrm{kg}$ and $1000 \mathrm{rad} / \mathrm{s}$, respectively, for each of the four sliding hyperplanes. The bound of the unbalance force is $9.52 \mathrm{~N}$ and only state 1 is assumed to have a non-zero initial condition upperbound. With this bound of $2.01 \mathrm{e}-04 \mathrm{~m}$, the upper bounds on $(X-\hat{X})$ are determined from Eq. (28) and the gains from Eq. (27). These gains are used in the numerical simulation of the state equations. From the simulation, the largest value of $(X-\hat{X})_{i}$ is determined and this value is compared to the value obtained analytically from Eq. (28). Inspection of Table II shows that the analytical upperbounds are greater than or equal to all the simulated values. The gain vector, $K_{\mathrm{v}}$, which is calculated off-line, is

$$
K_{\mathrm{v}}=\left[\begin{array}{llll}
42.81 & 32.69 & 42.81 & 32.69
\end{array}\right]^{\mathrm{T}} \mathrm{N} / \mathrm{kg} .
$$

TABLE II Upper bounds of $|X-\hat{X}|$ for $\Omega=6963 \mathrm{rpm}, \lambda=1000 \mathrm{rad} / \mathrm{s}$

\begin{tabular}{ccc}
\hline State $i$ & Analytical & Simulated \\
\hline 1 & $2.01 \mathrm{e}-04 \mathrm{~m}$ & $2.01 \mathrm{e}-04 \mathrm{~m}$ \\
2 & $8.34 \mathrm{e}-12 \mathrm{~m}$ & $6.02 \mathrm{e}-12 \mathrm{~m}$ \\
3 & $6.59 \mathrm{e}-05 \mathrm{~m}$ & $5.70 \mathrm{e}-05 \mathrm{~m}$ \\
4 & $5.92 \mathrm{e}-12 \mathrm{~m}$ & $5.03 \mathrm{e}-12 \mathrm{~m}$ \\
5 & $6.81 \mathrm{e}-05 \mathrm{~m}$ & $5.80 \mathrm{e}-05 \mathrm{~m}$ \\
6 & $9.41 \mathrm{e}-05 \mathrm{~m}$ & $6.93 \mathrm{e}-05 \mathrm{~m}$ \\
7 & $5.91 \mathrm{e}-02 \mathrm{~m} / \mathrm{s}$ & $5.38 \mathrm{e}-02 \mathrm{~m} / \mathrm{s}$ \\
8 & $5.16 \mathrm{e}-09 \mathrm{~m} / \mathrm{s}$ & $3.88 \mathrm{e}-09 \mathrm{~m} / \mathrm{s}$ \\
9 & $4.39 \mathrm{e}-02 \mathrm{~m} / \mathrm{s}$ & $3.73 \mathrm{e}-02 \mathrm{~m} / \mathrm{s}$ \\
10 & $3.91 \mathrm{e}-09 \mathrm{~m} / \mathrm{s}$ & $3.34 \mathrm{e}-09 \mathrm{~m} / \mathrm{s}$ \\
11 & $4.49 \mathrm{e}-02 \mathrm{~m} / \mathrm{s}$ & $3.86 \mathrm{e}-02 \mathrm{~m} / \mathrm{s}$ \\
12 & $5.89 \mathrm{e}-02 \mathrm{~m} / \mathrm{s}$ & $4.46 \mathrm{e}-02 \mathrm{~m} / \mathrm{s}$ \\
\hline
\end{tabular}

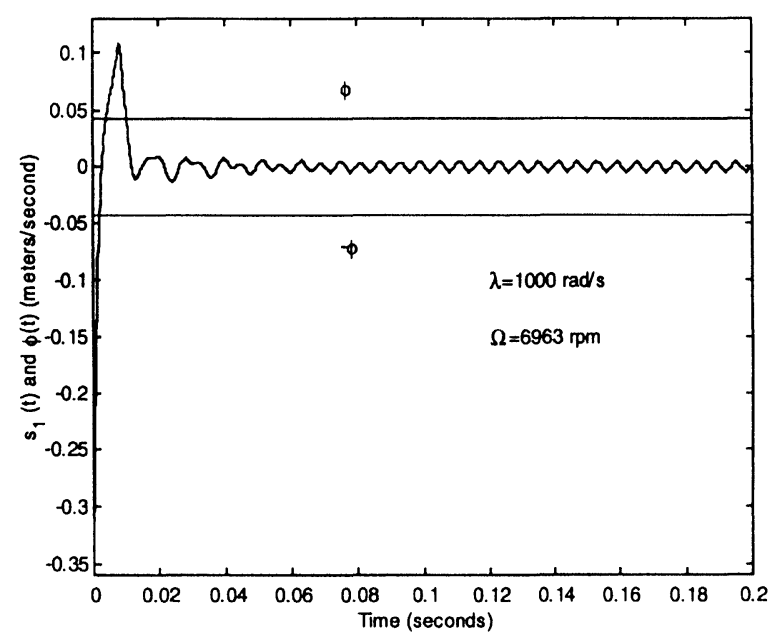

FIGURE $2 s_{1}$ and $\phi$ vs. time for $\Omega=6963 \mathrm{rpm}$.

\section{Steady-State Response}

Having established the validity of the approach for determining the control gains, it is desired to ascertain the effects of these gains as well as the control parameter, $\lambda$, on the response of the closed-loop system. Figure 2 shows a plot of $s_{1}(t)$ versus time of the rotor for an operating speed of $6963 \mathrm{rpm}$ resulting from the integration of Eqs. (24) and (25) where $\phi$ is the boundary layer thickness. Inspection of Fig. 2 indicates that the system remains inside the boundary layer after $0.01 \mathrm{~s}$ and thus can be described by the following set of linear equations:

$$
\dot{V}=A_{\text {sys }} V+D_{\text {sys }} F_{\mathrm{d}}(t)
$$


where

$$
\begin{gathered}
V=\left[\begin{array}{ccc}
X & \hat{X} & Z
\end{array}\right]^{\mathrm{T}} \\
A_{\mathrm{sys}}=\left[\begin{array}{ccc}
\tilde{A} & \tilde{B} \bar{P} C^{*} A & \tilde{B} K_{\mathrm{G}}^{\prime} \\
0 & \tilde{A}+\tilde{B} \bar{P} C^{*} A & \tilde{B} K_{\mathrm{G}}^{\prime} \\
\bar{P} C^{*} A & -\bar{P} C^{*} A & -K_{\mathrm{G}}^{\prime}
\end{array}\right] \\
D_{\mathrm{sys}}=\left[\begin{array}{c}
D \\
0 \\
\bar{P} C D
\end{array}\right] \\
K_{G}^{\prime}=\operatorname{diag}\left(\frac{k_{1}}{\phi_{1}}, \frac{k_{2}}{\phi_{2}}, \frac{k_{3}}{\phi_{3}}, \frac{k_{4}}{\phi_{4}}\right)
\end{gathered}
$$

The steady-state amplitude of $y_{1}(t)$ under the conditions above is $5.77 \mathrm{e}-06 \mathrm{~m}$. At the center of the shaft, where the rotor is located, the steady-state amplitude is $9.55 \mathrm{e}-05 \mathrm{~m}$. The system is stable if the eigenvalues of $A_{\text {sys }}$ lie in the left half of the complex plane. Stability issues in the sense of Lyapunov are discussed by Lewis and Sinha (1999). It has been shown that the eigenvalues of $A_{\text {sys }}$ are the eigenvalues of $\tilde{A}, \tilde{A}+\tilde{B} \bar{P} C^{*} A$, and $-K_{\mathrm{G}}^{\prime}$. The matrix $-K_{G}^{\prime}$ is guaranteed to be stable since it is always a diagonal matrix with negative elements. For colocated sensors and actuators, $\tilde{A}$ is stable. The remaining matrix, $\tilde{A}+\tilde{B} \bar{P} C^{*} A$, is stable if the symmetric parts of two symmetrizable matrices are positive definite.

Since the forcing function $F_{\mathrm{d}}(t)$ is sinusoidal in nature, a steady-state solution to Eq. (30) can be assumed as

$$
V=A_{11} \cos \Omega t+B_{11} \sin \Omega t
$$

where $A_{11}$ and $B_{11}$ are vectors of unknown elements. These vectors can be determined by substituting Eq. (35) into Eq. (30) and equating coefficients of $\sin \Omega t$ and $\cos \Omega t$. The first 12 elements of $V$ contain the state vector $X$; hence, the magnitude of the $i$ th steady-state output $Y_{i}$ is

$$
Y_{s s_{i}}=\sqrt{\left(\sum_{j=1}^{12} C_{i j}^{*} A_{11_{j}}\right)^{2}+\left(\sum_{j=1}^{12} C_{i j}^{*} B_{11_{j}}\right)^{2}}
$$

where $A_{11_{j}}$ and $B_{11_{j}}$ are the $j$ th elements of $A_{11}$ and $B_{11}$, respectively.

\section{Results of Parametric Study}

In the rotor/magnetic bearing system under study in this paper, there are four independent control inputs; i.e., there are effectively four actuators. It is assumed that each of these actuators has the same characteristics and that the bias currents (Appendix) $I_{0}$ and $I_{\mathrm{b}}$ are the same for each magnet pair. Furthermore, there are no parametric uncertainties associated with the magnet parameters. It is assumed that the value of $\lambda_{i}$ is the same for each of the four $s_{i}$ and that the initial conditions are the same for every analysis. Both $\lambda_{i}$ and $k_{i}$ influence the overall stiffness and damping of the system and thus the frequency response. As with any rotating machinery, the steady-state displacement is one of the chief concerns. And in the case of magnetic bearings, the current necessary to drive the actuator to achieve a desired displacement level is also an important issue. Since each bearing has the same characteristics and the value of each of the four $\lambda_{i}$ is the same, in the analyses that follow, the relationships that exist among the steady-state displacement $\left(y_{i}\right), \lambda_{i}, k_{i}, \Omega$, and incremental current will be presented for only one actuator.

The control law as expressed in Eq. (21) is dependent on two main parameters: the $4 \times 1$ vector of design variables, $\lambda_{i}$, and the $4 \times 1$ gain vector $K_{\mathrm{v}}$ that insures robustness to transient disturbances and external excitation. Inspection of Eqs. (27) and (28) indicates that $K_{\mathrm{v}}$ is directly proportional to the magnitude of the bound on the initial conditions. Consequently, a tighter bound estimate of these initial conditions will result in a smaller $K_{\mathrm{v}}$ which is important since the amount of current required to drive the actuators is dependent on $K_{\mathrm{v}}$. It is observed that $K_{\mathrm{v}}$ is directly influenced by the dynamic force $\left(\rho e \Omega^{2}\right)$ of the rotor since this term is in $F_{\mathrm{d}}(t)$ and $F_{\mathrm{d}}(t)$ is in the second term of Eq. (27). This is illustrated in Fig. 3, which shows how the gain $k_{1}$ (the first element of $K_{\mathrm{v}}$ ) varies as a function of the angular speed $\Omega$ for several fixed values of $\lambda_{1}$. 


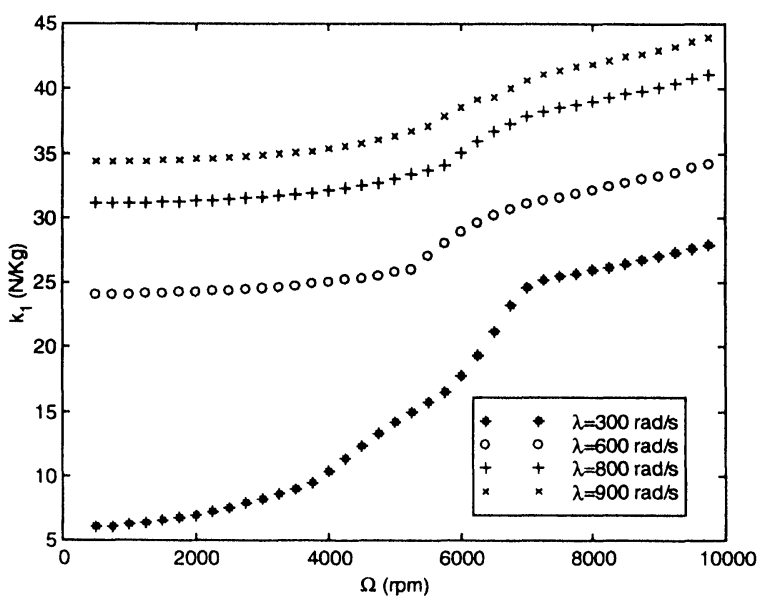

FIGURE $3 k_{1}$ vs. $\Omega$ for various values of $\lambda$.

Inspection of Fig. 3 indicates that $k_{1}$ increases gradually as a function of $\Omega$. This increase is more pronounced for the smaller values of $\lambda_{1}$ and in the region between 5000 and $6000 \mathrm{rpm}$. These values of $\Omega$ are directly related to the natural frequencies of the closed-loop system. Recalling that the openloop system had a critical frequency of $6963 \mathrm{rpm}$, it can be established that the design parameter $\lambda_{1}$ essentially causes this frequency to be shifted. Furthermore, $k_{1}$ increases as $\lambda_{1}$ increases. Although $\lambda$ does not appear explicitly in Eq. (27), its effects are included in the $\tilde{A}$ matrix of Eq. (28). Finally, there is some freedom of choice in that $K_{\mathrm{v}}$ is dependent on $\eta_{i}$, which can be selected by the designer, but are usually taken to be small positive constants.

Figure 4 shows a plot of $y_{1}$ at steady state versus $\Omega$ for various values of $\lambda_{1}$. It is evident that the magnitude of the steady-state displacement decreases steadily as $\lambda_{1}$ increases. This is further emphasized by Fig. 5, which shows the steady-state displacement as a function of $\lambda_{1}$ for several fixed values of $\Omega$. The magnitudes are noticeably higher for smaller values of $\lambda_{1}$ at $\Omega=7000 \mathrm{rpm}$ than those at other angular speeds. As $\lambda_{1}$ increases, the magnitude is highest for $\Omega=6000 \mathrm{rpm}$. This can be understood by realizing that the operating speed of $7000 \mathrm{rpm}$ is very close to the original open-loop

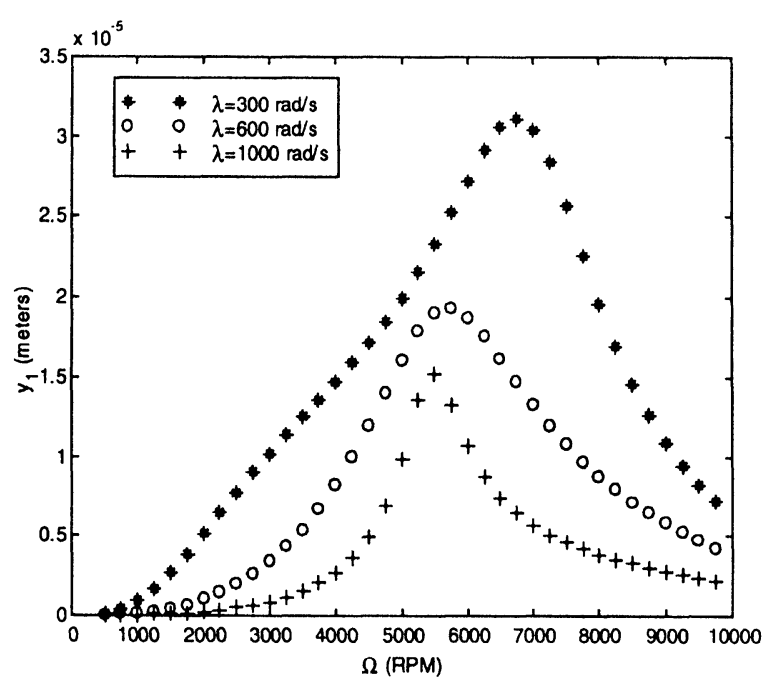

FIGURE $4 \quad y_{1}(t)$ vs. $\Omega$ at steady state.

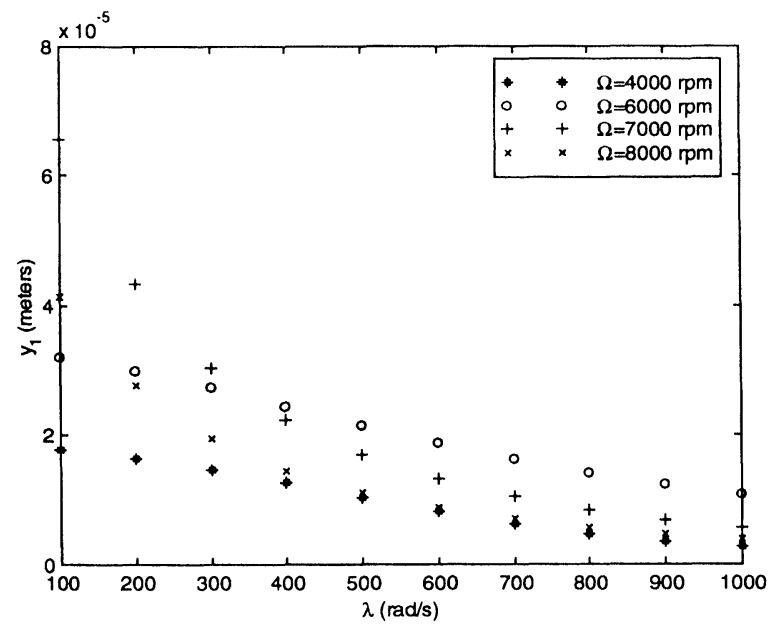

FIGURE $5 y_{1}(t)$ vs. $\lambda$ at steady state.

critical speed of $6963 \mathrm{rpm}$, and the shift in the critical speed is small for small values of $\lambda_{1}$. As $\lambda_{1}$ increases, there is a pronounced frequency shift from the open-loop critical frequency. This can be seen in Fig. 4 where it is observed the maximum steady-state value does not occur at the same angular speed for each $\lambda_{1}$.

In the analysis of rotating machinery, it is desirable to indicate the performance as it relates 


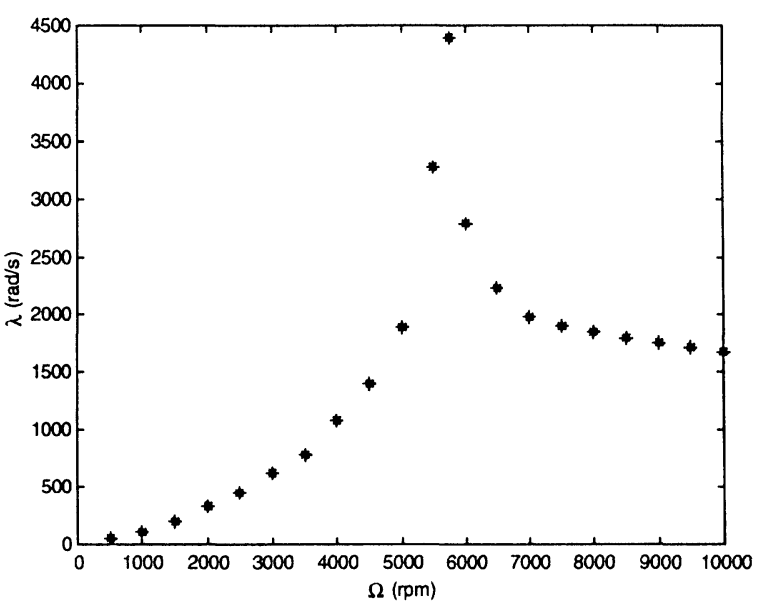

FIGURE $6 \lambda$ for good performance vs. $\Omega$.

to peak vibration levels. Figure 6 shows the values of $\lambda_{1}$ as a function of $\Omega$ needed to achieve "good" performance with regard to levels of vibration at the bearing locations as presented in the general machinery vibration severity chart, Rao (1995). Inspection of Fig. 6 shows that $\lambda_{1}$ increases as $\Omega$ increases until it reaches a maximum in the vicinity of $5750 \mathrm{rpm}$ and then decreases steadily which is consistent with the natural frequency of the closedloop system occurring at approximately $5750 \mathrm{rpm}$. Results from simulations indicate that the first flexible mode does not greatly impact the system response at the bearing locations. Table III shows the eigenvalues and their damping levels for both the open-loop $A$ matrix from Eq. (9) and the closedloop $\tilde{A}$ matrix from Eq. (26) for $\lambda=1000 \mathrm{rad} / \mathrm{s}$ and $\Omega=5750 \mathrm{rpm}$. It is observed that the effective damping ratio has been increased from 0.0113 to 0.074 for backward whirl and 0.0113 to 0.0831 for the forward whirl. These damping levels are a function of $\lambda$ and affect the behavior of the rotor system at all locations along the shaft. It has been found that values of $\lambda$ between 400 and $1000 \mathrm{rad} / \mathrm{s}$ produce higher damping ratios than values greater than $1000 \mathrm{rad} / \mathrm{s}$. As a result, a trade-off between effective damping levels (lower values of $\lambda$ ) in flexible modes and small displacements at the bearing locations (higher values of $\lambda$ (Figs. 4
TABLE III Eigenvalues of $A$ and $\tilde{A}$ for $\lambda=1000 \mathrm{rad} / \mathrm{s}$ and $\Omega=5750 \mathrm{rpm}$

\begin{tabular}{lccc}
\hline $\begin{array}{l}\text { Eigenvalues } \\
\text { of } A\end{array}$ & $\begin{array}{c}\text { Damping } \\
\text { ratio } \zeta\end{array}$ & $\begin{array}{c}\text { Eigenvalues } \\
\text { of } \tilde{A}\end{array}$ & $\begin{array}{c}\text { Damping } \\
\text { ratio } \zeta\end{array}$ \\
\hline 0.00 & 0.0 & $-871.00+j 114.00$ & 0.992 \\
0.00 & 0.0 & $-871.00-j 114.00$ & 0.992 \\
0.00 & 0.0 & $-955.00+j 408.00$ & 0.920 \\
0.00 & 0.0 & $-955.00-j 408.00$ & 0.920 \\
0.00 & 0.0 & $-974.00+j 426.00$ & 0.916 \\
0.00 & 0.0 & $-974.00-j 426.00$ & 0.916 \\
$+j 33.80$ & 0.0 & $-1130.00+j 147.00$ & 0.992 \\
$-j 33.80$ & 0.0 & $-1130.00-j 147.00$ & 0.992 \\
$-8.27+j 734.00$ & 0.0113 & $-41.10+j 548.00$ & 0.0747 \\
$-8.27-j 734.00$ & 0.0113 & $-41.10-j 548.00$ & 0.0747 \\
$-8.70+j 771.00$ & 0.0113 & $-47.30+j 568.00$ & 0.0831 \\
$-8.70-j 771.00$ & 0.0113 & $-47.30-j 568.00$ & 0.0831 \\
\hline
\end{tabular}

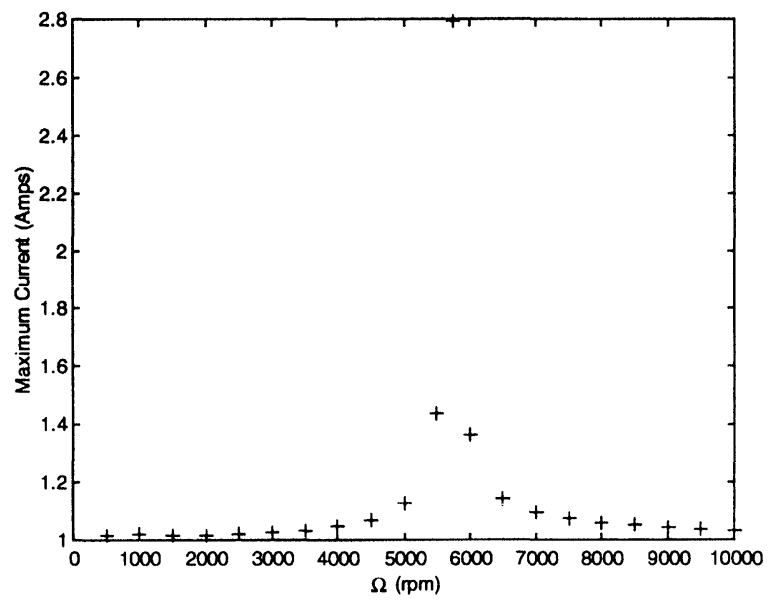

FIGURE 7 Maximum current $I_{11}$ vs. $\Omega$ for $\lambda$ for good performance.

and 5)) must be made. The zero eigenvalues of the $A$ matrix represent the rigid body modes indicative of the free-free open-loop rotor system. By constraining the system to stay on the sliding manifold, the sliding mode controller in essence shifts the eigenvalues and renders the mode shapes of the rotor system similar to those with fixed-fixed boundary conditions.

Figure 7 shows a plot of the magnitude of the maximum current (Appendix) versus $\Omega$ in steady state corresponding to the values of $\lambda$ for good 
performance as shown in Fig. 6. The maximum value of the current occurs at approximately $5750 \mathrm{rpm}$ which is near the closed-loop natural frequency. This peak value of total current in the top magnet is approximately $2.8 \mathrm{~A}$. The bias current, $I_{0}$, is $1.0 \mathrm{~A}$ for all $\Omega$ except $\Omega=5750 \mathrm{rpm}$. The bias current has been changed for $5750 \mathrm{rpm}$ to $1.8 \mathrm{~A}$ to ensure that the total current is always positive. Figure 7 also indicates that the required incremental current may be large in the area of the closed-loop critical frequency and raises the possibility that the linearized law for current computation may not be valid in this region. As a result, a non-linear approach has also been developed (Appendix). It has been found that both the linear and non-linear approaches yield virtually the same incremental current.

\section{SUMMARY AND CONCLUSIONS}

This paper deals with the active vibration control of a flexible rotor via magnetic bearings using only output feedback. A novel sliding mode algorithm to control the rotor robustly in the event of transient disturbances and external excitation has been presented. It is shown that the required gain vector can be calculated easily off-line for the known bounds of rotor unbalance.

Numerical results demonstrate the validity of this novel control algorithm. Results from parametric studies are also presented. In particular, control parameters required for "good" performance are presented as a function of the angular speed of the rotor. Only the uncertainty in the rotor unbalance has been considered in this paper. Future work will be aimed towards parametric errors in the rotor model and magnetic bearings, and high-frequency unmodeled dynamics.

\section{Acknowledgement}

This work was supported in part by grant NAGW1356 from the Propulsion Engineering Research Center at the Pennsylvania State University.

\section{NOMENCLATURE}

\begin{tabular}{|c|c|}
\hline$a, b$ & Location of sensors along rotor \\
\hline$e^{\prime}$ & $\begin{array}{l}\text { Distance between geometric center } \\
\text { of the rotor and overall rotor mass } \\
\text { center }\end{array}$ \\
\hline$e$ & Rotor eccentricity \\
\hline$h_{0}$ & Airgap \\
\hline$m_{\mathrm{t}}, m_{\mathrm{d}}$ & $\begin{array}{l}\text { Total mass of rotor system and } \\
\text { mass of main disk, respectively }\end{array}$ \\
\hline$p_{i}, q_{i}$ & Generalized coordinates \\
\hline$r$ & Full order coordinate vector \\
\hline$A_{\mathrm{p}}$ & Face area per single pole of magnet \\
\hline$A_{\text {sys }}, D_{\text {sys }}$ & $\begin{array}{l}\text { Overall system state and } \\
\text { disturbance matrices, respectively }\end{array}$ \\
\hline$A, B, D, C^{*}$ & $\begin{array}{l}\text { Reduced order state, control, } \\
\text { disturbance, and output matrices, } \\
\text { respectively }\end{array}$ \\
\hline $\bar{B}, \bar{D}$ & $\begin{array}{l}\text { Control and disturbance matrices, } \\
\text { respectively }\end{array}$ \\
\hline$C_{\mathrm{D}}$ & $\begin{array}{l}\text { Constant selected to provide a } \\
\text { specified damping ratio }\end{array}$ \\
\hline$F_{\mathrm{c}}, F_{\mathrm{d}}(t)$ & $\begin{array}{l}\text { Control force vector and } \\
\text { disturbance force vector, } \\
\text { respectively }\end{array}$ \\
\hline$I_{\mathrm{b}}, I_{0}$ & $\begin{array}{l}\text { Bias currents in top and bottom } \\
\text { magnets, respectively }\end{array}$ \\
\hline$K_{\mathrm{G}}$ & $\begin{array}{l}\text { Diagonal matrix of control gains } \\
\left(k_{1}, k_{2}, k_{3}, k_{4}\right)\end{array}$ \\
\hline$L$ & Length of rotor \\
\hline $\bar{M}, \bar{G}, \bar{K}$ & $\begin{array}{l}\text { Mass, damping/gyroscopic, and } \\
\text { stiffness matrices, respectively }\end{array}$ \\
\hline$N_{\mathrm{p}}$ & Number of coil turns \\
\hline$P, P_{1}, \bar{P}, P^{*}$ & $\begin{array}{l}\text { Various matrices used in the } \\
\text { control law }\end{array}$ \\
\hline$S$ & $\begin{array}{l}\text { Vector of sliding hyperplanes } \\
\left(s_{1}, s_{2}, s_{3}, s_{4}\right)\end{array}$ \\
\hline $\bar{S}^{\prime}$ & Vector of saturation functions \\
\hline$U$ & Control law \\
\hline$V$ & Overall system vector \\
\hline$X_{1}, X_{2}$ & $\begin{array}{l}\text { Components of reduced order state } \\
\text { vector } X\end{array}$ \\
\hline$\hat{X}$ & Observer state vector \\
\hline$\tilde{A}, \tilde{B}$ & $\begin{array}{l}\text { Closed loop state and control } \\
\text { matrices, respectively }\end{array}$ \\
\hline
\end{tabular}




$\begin{array}{ll}Y_{1}, Y_{2} & \begin{array}{l}\text { Position }\left(y_{1}, y_{2}, y_{3}, y_{4}\right) \text { and velocity } \\ \left(\dot{y}_{1}, \dot{y}_{2}, \dot{y}_{3}, \dot{y}_{4}\right) \text { output vectors, } \\ \end{array} \\ & \begin{array}{l}\text { respectively } \\ \text { Vector used in control law }\end{array} \\ & \text { containing integral of output } \\ \lambda_{i} & \text { The } i \text { th control design parameter } \\ \zeta & \text { Reduced-order coordinate vector } \\ \rho & \text { Mass of unbalance } \\ \eta & \text { Small positive constant for } \\ & \text { control law } \\ \mu_{0} & \text { Permeability of free space } \\ \Phi & \text { Mass normalized eigenvector matrix } \\ \Omega & \text { Angular speed of rotor }\end{array}$

\section{References}

Allaire, P.E., Humphris, R.R. and Barrett, L.E., 1986. Critical speeds and unbalance response of a flexible rotor in magnetic bearings, Proceedings of the First European Turbomachinery Symposium, London, pp. 111-120.

Asada, H. and Slotine, J.J.E., 1986. Robot Analysis and Control, John Wiley and Sons, New York.

Bobbert, M.F. and Schamhardt, H.C., 1990. Accuracy of determining the point of force application with piezoelectric force plates, Journal of Biomechanics, 23(7), 705-710.

Dimarogonas, A.D. and Paipetis, S.A., 1983. Analytical Methods in Rotordynamics, Applied Science Publishers, London, New York.

Herzog, R., Bühler, P., Gähler, C. and Larsonneur, R., 1996. Unbalance compensation using generalized notch filters in the multivariable feedback of magnetic bearings, IEEE Transactions on Control Systems Technology, 4(5), September 1996, $580-586$.

Humphris, R.R., Kelm, R.D., Lewis, D.W. and Allaire, P.E., 1986. Effect of control algorithms on magnetic journal bearing properties, ASME Paper No. 86-GT-54.

Knospe, C.R. and Tamer, S.M., 1997. Robust adaptive control of unbalance response for a flexible rotor, JSME International Journal Series C, 40(4), 599-606.

LaLanne, M. and Ferraris, G., 1990. Rotordynamics Prediction in Engineering, John Wiley and Sons, Chichester, England.

Lewis, A.S., 1994. Sliding mode control of a flexible rotor via magnetic bearings: Theory and experiments, $\mathrm{Ph} . \mathrm{D}$. Thesis, The Pennsylvania State University, University Park, PA.

Lewis, A.S. and Sinha, A., 1995. Sliding mode output feedback control of mechanical systems with bounded disturbances, Proceedings of the 1995 American Control Conference, Seattle, Washington, pp. 3735-3739.

Lewis, A.S. and Sinha, A., 1999. Sliding mode control of mechanical systems with bounded disturbances via output feedback, Journal of Guidance, Control, and Dynamics, 22(2), 235-240.

Lum, K.Y., Coppola, V.T. and Bernstein, D.S., 1996. Adaptive autocentering control for an active magnetic bearing supporting a rotor with unknown mass imbalance, IEEE Transactions on Control Systems Technology, 4(5), September 1996, 587-597.
Nonami, K. and Ito, T., 1996. $\mu$-Synthesis of flexible rotormagnetic bearing systems, IEEE Transactions on Control Systems Technology, 4(5), 503-512.

Okada, Y., 1994. Disturbance observer based controller for flexible rotor supported by magnetic bearings, Fourth International Symposium on Magnetic Bearings, August 1994, ETH Zurich.

Palazzolo, A.B., Lin, R.R., Kascak, A.F., Montague, J. and Alexander, R.M., 1989. Test and theory for piezoelectric actuator-active vibration control of rotating machinery, Rotating Machinery Dynamics, ASME, DE-18(1), 367-374.

Rao, S.S., 1995. Mechanical Vibrations, Addison-Wesley Publishing Company, Inc., p. 567.

Rundell, A.E., Drakunov, S.V. and DeCarlo, R.A., 1996. A sliding mode observer and controller for stabilization of rotational motion of a vertical shaft magnetic bearing, IEEE Transactions on Control Systems Technology, 4(5), 598-608.

Salm, J. and Schweitzer, G., 1984. Modeling and control of a flexible rotor with magnetic bearings, Proceedings of Third International Conference on Vibrations in Rotating Machinery, University of York, England, pp. 553-559.

Sinha, A., 1990. On the design of magnetic suspension systems, ASME Paper No. 90-GT-239.

Sinha, A., Meese, K.L. and Wang, K.W., 1991. Sliding mode control of a rigid rotor via magnetic bearings, ASME Biennial Conference on Mechanical Vibration and Noise, Miami, Florida.

Tian, H. and Nonami, K., 1996. Discrete-time sliding mode control of flexible rotor-magnetic bearing systems, International Journal of Robust and Nonlinear Control, 6, 609-632.

Ulbrich, H. and Anton, E., 1984. Theory and application of magnetic bearings with integrated displacement and velocity sensors, IMechE, pp. 543-551.

Utkin, V., 1977. Variable structure systems with sliding modes, IEEE Transactions on Automatic Control, AC-22, 212-222.

Utkin, V., 1983. Variable structure systems: Present and future, Automation and Remote Control, 44(9), 1105-1119.

Vance, J.M., 1988. Rotordynamics of Turbomachinery, John Wiley and Sons, New York.

Wang, W.-J. and Fan, Y.-T., 1994. New output feedback design in variable structure system, AIAA Journal of Guidance, Control, and Dynamics, 17(2), 337-340.

Yallapragada, S.V., Heck, B.S. and Finner, J.D., 1996. Reaching conditions for variable structure control with output feedback, AIAA Journal of Guidance, Control and Dynamics, 19(4), $848-853$.

\section{APPENDIX (COMPUTATION OF COIL CURRENTS)}

It is necessary to relate the control force, Eqs. (20) and (21), to the desired current to be delivered by magnetic bearings. Figure 1 shows a schematic of the important parameters involved in the calculation of the coil currents. $F_{a x}$, the net force on the left end of the shaft in the 
$x$-direction, can be written as

$$
\begin{aligned}
F_{a x}= & H \frac{\left(I_{\mathrm{b} 2}+\hat{I}_{11}\right)^{2}}{\left(h_{0}-y_{1}\right)^{2}}-H \frac{\left(I_{0}+\hat{I}_{12}\right)^{2}}{\left(h_{0}+y_{1}\right)^{2}} \\
& -\left(\frac{l_{2}}{l_{1}+l_{2}}\right) m_{\mathrm{t}} g \sin \theta
\end{aligned}
$$

where $h_{0}$ is the nominal airgap at equilibrium, $m_{\mathrm{t}}$ the total mass of the rotor system, $g$ the acceleration due to gravity, and $H=\mu_{0} A_{\mathrm{p}} N_{\mathrm{p}}^{2}$ is a constant associated with the electromagnets. Here, $N_{\mathrm{p}}, A_{\mathrm{p}}$, and $\mu_{0}$ are the number of coil turns, the face area per single pole of magnet, and the permeability of free space, respectively. Similar equations can be established for $F_{a y}, F_{b x}$, and $F_{b y}$. In Eq. (A.1), the first and second terms on the right hand side represent the attractive forces of the top and bottom magnets, respectively. The last term accounts for the weight of the rotor system. $I_{\mathrm{b} 2}$ and $I_{0}$ represent the bias currents in the top and bottom magnets under static equilibrium conditions. The incremental currents of the top and bottom magnets can be either positive or negative and are denoted $\hat{I}_{11}$ and $\hat{I}_{12}$, respectively. The axes of the bearing actuators are located at an angle $\theta$ from the vertical. The bias current $I_{\mathrm{b} 2}$ is calculated as

$$
I_{\mathrm{b} 2}=\frac{h_{0}}{\sqrt{H}} \sqrt{\frac{H I_{0}^{2}}{h_{0}^{2}}+\left(\frac{l_{2}}{l_{1}+l_{2}}\right) m_{\mathrm{t}} g \sin \theta}
$$

\section{LINEAR APPROACH FOR INCREMENTAL CURRENT CALCULATION}

Linearizing the control force, Eq. (A.1) about the bias currents $I_{0}$ and $I_{\mathrm{b} 2}$ and the nominal airgap, $h_{0}$, with

$$
F_{a x}=\left(\frac{2 H I_{\mathrm{b} 2}+2 H I_{0}}{h_{0}^{2}}\right) \hat{I}_{11}+\left(\frac{2 H I_{\mathrm{b} 2}^{2}+2 H I_{0}^{2}}{h_{0}^{2}}\right) \frac{y_{1}}{h_{0}}
$$

where $\hat{I}_{12}=-\hat{I}_{11}$. The incremental current in the top magnets $\left(\hat{I}_{11}\right)$ is obtained from Eq. (A.3) and the total coil current for the pair of magnets is

$$
\begin{aligned}
& I_{11}=I_{\mathrm{b} 2}+\hat{I}_{11} \\
& I_{12}=I_{0}+\hat{I}_{12}
\end{aligned}
$$

Since the incremental current and force are linearly related, an equivalent approach would also be to modify Eq. (8) such that the control law would yield the incremental current directly.

\section{Non-linear Approach for Incremental Current Calculation}

Sinha (1990) has computed the incremental current without linearization as

$$
\hat{I}_{11}=\frac{-a_{2}+\sqrt{a_{2}^{2}-4 a_{1} a_{3}}}{2 a_{1}}
$$

where

$$
\begin{aligned}
a_{1}= & \frac{H}{\left(h_{0}-y_{1}\right)^{2}}-\frac{H}{\left(h_{0}+y_{1}\right)^{2}} \\
a_{2}= & \frac{2 H I_{\mathrm{b} 2}}{\left(h_{0}-y_{1}\right)^{2}}+\frac{2 H I_{0}}{\left(h_{0}+y_{1}\right)^{2}} \\
a_{3}= & \frac{H I_{\mathrm{b} 2}^{2}}{\left(h_{0}-y_{1}\right)^{2}}-\frac{H I_{0}^{2}}{\left(h_{0}+y_{1}\right)^{2}} \\
& -\frac{l_{2}}{l_{1}+l_{2}} m_{\mathrm{t}} g \sin \theta-F_{a x}
\end{aligned}
$$

The total current is given by Eqs. (A.4) and (A.5) where $\hat{I}_{12}=-\hat{I}_{11}$. 

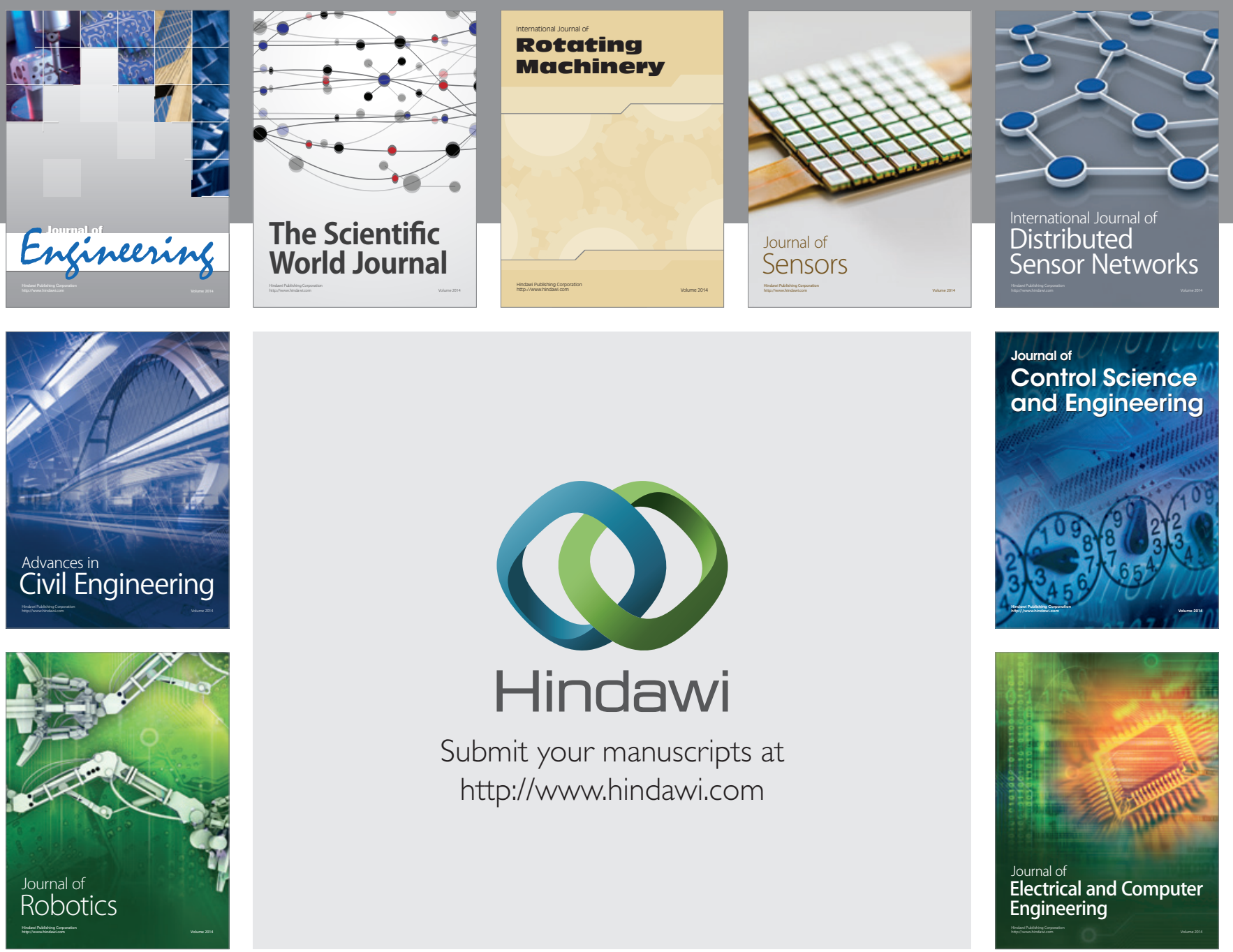

Submit your manuscripts at

http://www.hindawi.com
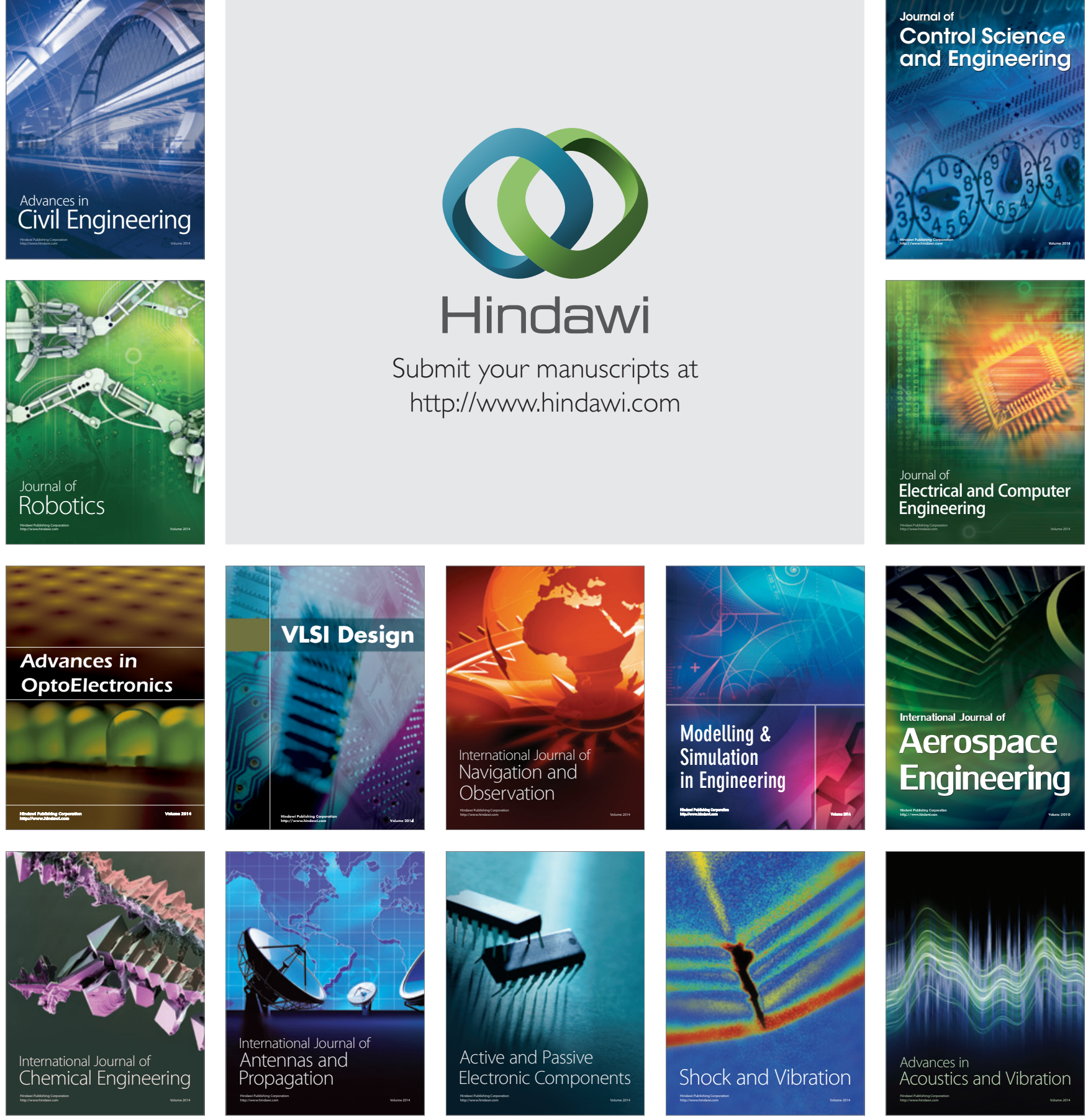